\title{
A Wavelet De-Noising Algorithm Based on Adaptive Threshold Function
}

\author{
Cao Wei* and Li Hui \\ Harbin University of Science and Technology, Harbin, 150080, P. R. China \\ 77274127@qq.com
}

\begin{abstract}
For the problems of traditional wavelet de-noising algorithm in dealing with non-stationary signals will destroy the detail component of signal. Paper proposes an adaptive wavelet de-noising algorithm. First, a simplified mathematical model of wavelet de-noising algorithm was established. Analyze the problem of traditional wavelet de-noising algorithm. Then, an adaptive threshold function mathematical model was established according to the problem of traditional wavelet de-noising algorithm. Determine the parameter by the relationships between different scales of wavelet decomposition of signal and noise. Finally, calculate the optimal threshold of the adaptive threshold function by iterative algorithm. Simulation results show that the adaptive wavelet threshold de-noising algorithm can preserve the original signal details. And adaptive wavelet threshold de-noising algorithm is better than the traditional wavelet de-noising algorithm in the signal to noise ratio, mean square error and so on.
\end{abstract}

Keywords: wavelet de-noising; digital signal process; adaptive threshold function; adaptive threshold

\section{Introduction}

Signals in the collection, transmission and A/D conversion easily mixed with noise. This can cause signal degradation and adversely affect the subsequent signal processing. Especially in the field which require signal quality. Especially in the field of higher requirements on signal quality, Such as Communications, sophisticated detection and speech recognition. In order to ensure that the signal recognition, feature analysis, the signal must be de-noising [1-2]. In practical engineering applications, de-noising requires not only suppress noise, it must ensure that does not destroy the signal detail component.

Signals can be divided into stationary signal and non-stationary signal. In practice, most signals are non-stationary signals [3]. Fourier transform cannot get the details of the non-stationary signals, so the Fourier transform cannot deal with non-stationary signals. The wavelet transform is developed on the basis of Fourier; wavelet transform uses wavelets instead of the sinusoidal basis in Fourier transform [4]. Therefore, the wavelet transform can extract the details of the signal; the wavelet transform can be used to handle non-stationary signals.

In recent years, with further research, wavelet analysis obtains a larger development in the field of signal de-noising. Wavelet De-noising algorithm can be divided into Maximum value de-noising algorithm. Correlation de-noising algorithm and Threshold de-noising algorithm. Because of its simple algorithm and small computation quantity, de-noising by thresholding obtained the widespread application [5]. However, there are some problems in the traditional wavelet threshold algorithm. Therefore, this paper constructs a new adaptive threshold function, and adopt iterative algorithm to obtain the optimal threshold in different decomposition scale.

${ }^{*}$ Corresponding Author 


\section{Wavelet Threshold De-Noising}

\subsection{Traditional De-Noising Algorithm}

When the signal de-noising, not only to consider how to remove the noise, but also should consider a issue that how to preserve partial the feature of signal, the problem will be more obvious when dealing with the signal which have low noise-signal ratio. In reality, it is often encountered that the signal is almost completely submerged in noise, such as fetal ECG and the signal generated by the propeller of submarine, etc. There singles with low noise-signal ratio, and carries a lot of detail component. Therefore, it is of great significance to study the de-noise algorithm of this kind of signal.

Figure 1 (a), is real output signal of Strain Gauge Transducer. As can be seen from the signal waveform, signal is mixed with a certain degree of noise in the process of acquisition and transmission. And the original signal carries a lot of mutation pikes, these mutations peaks can be seen as details of the signal, these details may carry some important information. Thus we need to preserve the details. Figure 1 (b), is a rendering which the soft-threshold de-noising algorithm is adopted to noise signal process. It can be seen that although the soft threshold algorithm can preserve the detail component of signal, but the effect is not desirable. Figure 1(c), is a renderings which the hard-threshold de-noising algorithm is adopted to noise signal process, it can be seen that the single may lack of details when the single processed by hard-threshold de-noise algorithm. Therefore, it is necessary to construct a de-noise algorithm that can satisfy de-noise and preserve signal details composition.
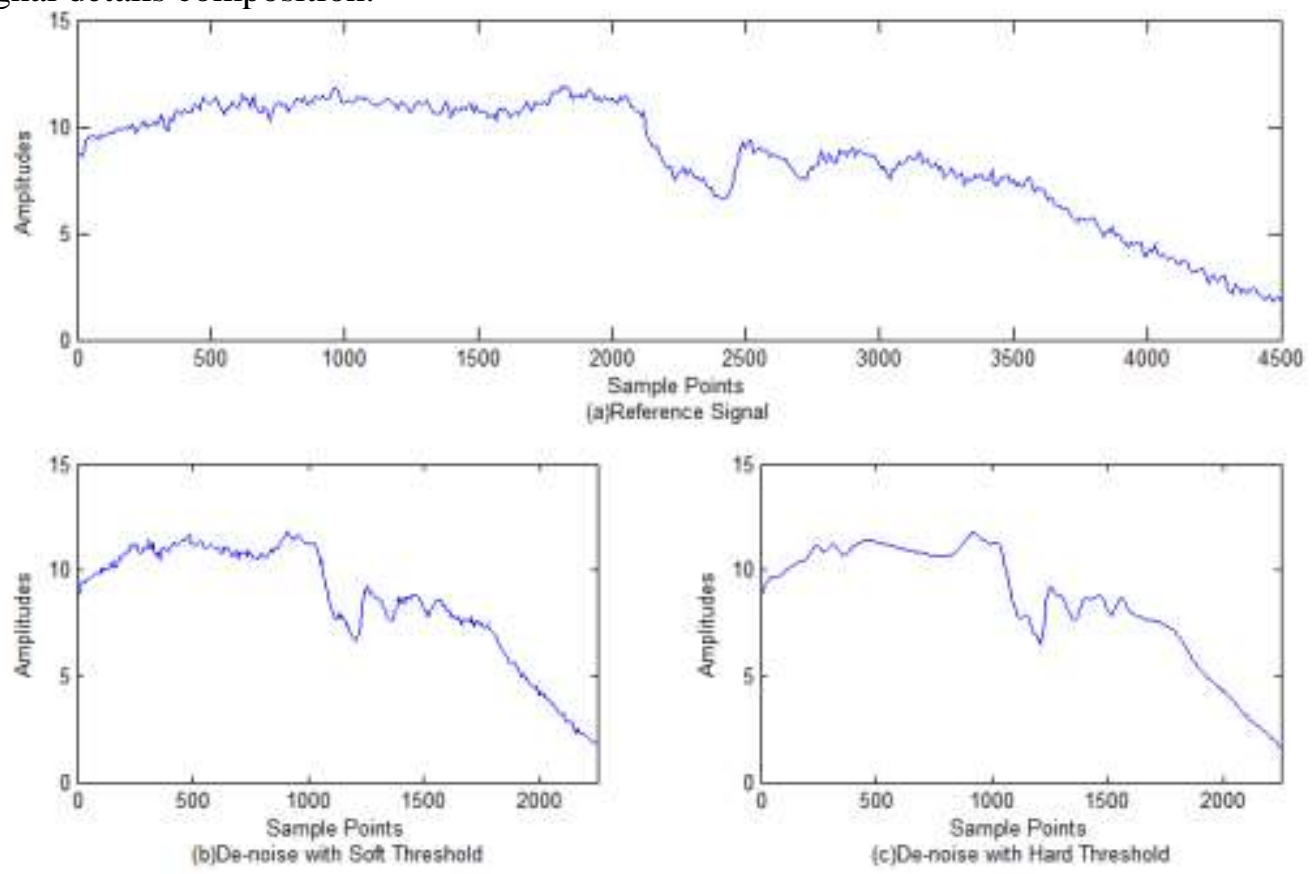

Figure 1. The Effect of Strain Gauge Sensor Output Signal Processed by Soft and Hard Threshold De-Noise Algorithm

\subsection{Wavelet Thresholding Principle and Mathematical Model}

The principle of wavelet threshold de-noising can be summarized as: the wavelet transform of the contaminated signal, signal components present only in a small number of wavelet coefficients, This indicates that the signal wavelet coefficients in the wavelet domain is a partial distribution, And these wavelet coefficients present in all wavelet scales; But the wavelet coefficients of noise present in all the range of wavelet domain, 
And the wavelet coefficients of the noise will be reduced significantly when the decomposition scale increases [8].

Therefore the wavelet de-noising process can be summarized as the following three steps:

(1)Wavelet transform to the contaminated signal.

(2)For the low-frequency signal components in maximum resolution scales, it can be seen as part of the real signal, so this part of the signal should be preserved; the high-frequency signal components of the respective scales can be seen as the true signal and noise superimposed, thus this part need to do nonlinear process.

(3)IWDT for wavelet coefficients, get the signal after de-noise.

For the signal with noise it can be represented as a mathematical model:

$y=x+n$

In the Formula (1), $y$ representative of the sequence of the contaminated signal, Which is composed of the signal $x$ and the noise $n$.

The three processes of wavelet de-noise can be described by a mathematical model:

$\left\{\begin{array}{l}u=W y) \\ \tilde{u}=\mathbb{Q} u, \text { t h }) \\ \hat{x}=W^{\prime}(\tilde{u})\end{array}\right.$

$W$ represents wavelet transform, $u$ is the wavelet coefficients obtained after wavelet transform; D represents de-noise operation to the wavelet coefficients $u$. $\tilde{u}$ is wavelet coefficients obtained by de-noise operation; $W$ is the inverse wavelet transform operation. $\hat{x}$ is the signal after de-noise operation. The critical procedure of the wavelet threshold de-noise is nonlinear de-noise algorithm, How to select the appropriate threshold function and find the corresponding threshold is a major problem of wavelet threshold de-noise algorithm.

\subsection{Self-Adaptive Threshold Functions}

In order to solve the problem of the traditional threshold de-noising algorithm, it is necessary to construct an appropriate threshold function. According to the threshold function selection principle of the wavelet threshold de-noise algorithm, an excellent threshold function need to satisfy the following characteristics:

(1)The threshold function is continuous in the wavelet domain.

(2)Threshold function should ensure the estimation of the mean square error (MSE) have minimum value.

(3)The threshold function should have first-order derivative or more.

Thus, this paper constructs an improved threshold function, parameters can be adjusted according to the intensity of the noise. Thereby adjusting the soft and hard degree of threshold function. The improved threshold function can adjust the soft and hard degree of the threshold function, which is a variable threshold function. The expression for the variable threshold function is:

$$
\eta(x, \mathrm{th}, m n)=\left\{\begin{array}{l}
x-0.5 \operatorname{sgn}(x) \frac{\mathrm{th}^{m}}{|x|^{m-1}},|x|>\mathrm{th} \\
0.5 \operatorname{sgn}(x) \frac{|x|^{n}}{\mathrm{th}^{n-1}}, \quad|x| \leq \mathrm{th}
\end{array}\right.
$$

In Formula (3), th is the threshold, $m$ and $n$ are adjustment parameters. In order to make the threshold function have first-order derivative, the condition should be satisfied: 
$\left.\frac{\partial \eta(x, \mathrm{th}, m n)}{\partial x}\right|_{x=\mathrm{th}^{-}}=\left.\frac{\partial \eta(x, \mathrm{th}, m n)}{\partial x}\right|_{x=\mathrm{th}^{+}}$

According to Formula (4), when $n=m+1$, the threshold function of the Formula (3), has the first-order derivative. Thus, the threshold function of the Formula (3), can be written as:

$\eta(x, \mathrm{th}, m)=\left\{\begin{array}{l}x-0.5 \operatorname{sgn}(x) \frac{\mathrm{th}^{m}}{|x|^{m-1}},|x|>\mathrm{th} \\ 0.5 \operatorname{sgn}(x) \frac{|x|^{m+1}}{\mathrm{th}^{m}}, \quad|x| \leq \mathrm{th}\end{array}\right.$

Figure 2, shows the corresponding threshold function image when the parameter $m$ is 1,5 and 10 .

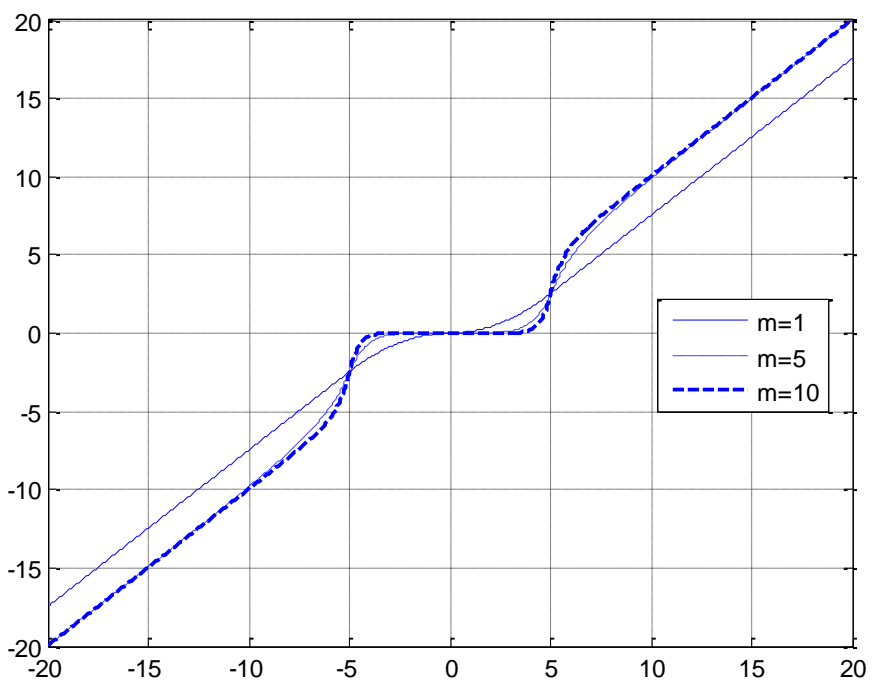

Figure 2. Variable Threshold Function

From the Figure 2, we can know that when $m=1$, the threshold function can be regarded as the soft threshold function; and when $m=10$, the curve of the threshold function is almost coincident with the hard threshold function curve. So we can select the most suitable threshold function $\eta^{j}(x, \mathrm{th}, m)$ in different decomposition scales by adjusting the parameters $m$. According to the energy distribution characteristics of signal and noise in different decomposition scales: the energy of the noise component gradually decreases with the increase of the decomposition scale. Therefore, the noise component is abounding in the fine scale, it should be used hard threshold function to process wavelet coefficients, this can effective inhibit the noise. But in the wide scale, the soft threshold function is used to prevent the details of the signal.

In order to adjust the threshold function, establish a function of the parameter $m$ as follows:

$$
m=10^{\frac{E_{n j}}{E_{d j}}}
$$

Wherein, $E_{d j}=\sum_{k=0}^{N-1} d_{j k}^{2}$ is the total energy of the signal in the wavelet decomposition scale $j . E_{n j}$ is the noise energy in the wavelet decomposition scale $j$. 
Find the relevant literature, the Gaussian noise has the following distribution characteristics in the wavelet scale $j$ :

(1)In scale $d_{1}$, the energy of the signal is approximately equal to the energy of the noise.

(2)The energy of white Gaussian noise in the wavelet scale $j$ is $2 \mathrm{j}$ of the total energy of the noise.

It can determine the energy of white Gaussian noise on wavelet scale $j$ is $E_{n j} \approx \frac{1}{2^{j}} E_{n} \approx \frac{1}{2^{j-1}} \sum_{k=0}^{N-1} d_{1 k}^{2}$

In summary, when the wavelet scale $j=1$, the noise energy can be reached to the maximum, at this time, $m$ has a maximum value, with the increase of wavelet decomposition scale, the energy of noise is gradually reduced, the value of $m$ also decreases. This can achieve the change of threshold function.

\section{The De-Noise Algorithm Based on Self-adapting Threshold Function}

\subsection{The Selection of the Optimal Threshold}

Universal threshold functions have proven to be one of the simplest ways in the field of wavelet de-noising. However, on account of the value of the universal-threshold equals to the upper limit of the wavelet shrinkage threshold some information of the input signal may be lost. Thus, It is necessary to select the optimal threshold of the actual signal. When the error of mean square between the measuring signals and the true signals is minimum, the optimal threshold will be determined. On the basics of the SURE(Stein Unbiased Risk Estimate), the unbiased estimate of the minimum mean square error can be obtained. The actual implementation is to estimate the value of actual signal the estimated value equals to $\tilde{x}$. Therefore the value actual signal $x$ can be replaced by $\tilde{x}$. Hence, it is effective to minimize the error of mean square between actual signal and the estimated value $R \tilde{x}, x)=E\|\tilde{x}-x\|^{2}$.

To determine the error of mean square $R \tilde{x}, x)$, it is need to process the measured value of actual signal. $y$ denotes measurement signal, $x$ denotes actual signal, $n$ denotes noise signal, $\hat{u}, u$ and $v$ respectively denote the wavelet coefficients. According to Formula (1), the mathematical model of wavelet domain can be obtained which is as following:

$\hat{u}=u+v$

Establish a mapping function:

$$
g(y)=\hat{f}(y)-y
$$

Where $\hat{f}(y)$ is the estimated value of $y$. Meanwhile, $\hat{f}(y)$ is the threshold function $\eta=(y, \mathrm{th}, m)$.

According to Formula (8), the following formula can be obtained.

$$
\begin{aligned}
E\|g(y)\|^{2} & =E\|\hat{f}(y)-y\|^{2}=E\|f(y)-(x+n)\|^{2} \\
& =E\|\hat{f}(y)-x\|^{2}+E\|n\|^{2}+0 \\
& =E\|\hat{u}-u\|^{2}+E\|v\|^{2}
\end{aligned}
$$


According to Parseval's theorem, the value of $E\|\hat{f}(y)-y\|^{2}$ can be minimized when the value of $E\|\hat{u}-u\|^{2}$ is the minimum. Meanwhile, as $g(y)$ is differentiable, formula (10) can be obtained on the basics of SURE theorem.

$E\|\hat{f}(y)-y\|^{2}=N+E\left[\|g(y)\|^{2}+2 \nabla_{y} \cdot g(y)\right]$

Thus, the mean square error equation can be obtained, which is Formula (11).

$R(t)=N+\|g(y)\|^{2}+2 \nabla_{y} \cdot g(y)$

\subsection{Adaptive De-Noise Algorithm}

The optimal threshold value can be obtained by solving the mean square error equation. Typically, the computer using Newton's method or the Steepest Descent method to implement the process of solving non-linear equations. Newton's method is two order convergences, and Steepest Descent method is first order convergence, so the time that solving non-linear equations with Newton's method is less then Steepest descent method. Therefore, this paper solves the MSE equation by Newton's method.

The iterative formulas of Newton's method as follow:

$\mathrm{th}_{k+1}=\mathrm{th}_{k}-\frac{R\left(\mathrm{th}_{k}\right)}{R\left(\mathrm{th}_{k}\right)}$

In the Formula (12), $t h_{k}$ is the threshold after $k$ times iterations, $R\left(h_{k}\right)$ is the MSE equation. $R\left(\mathrm{t} \mathrm{h}_{k}\right)$ is the first derivative for $\left.R \mathrm{t} \mathrm{h} k\right)$.

$$
\begin{aligned}
R\left(\mathrm{th}_{k}\right) & =\frac{\left.\partial \boldsymbol{R} \mathrm{th}_{k}\right)}{\partial \mathrm{th}_{k}} \\
& =2 \sum_{i=0}^{N-1} g_{i} \cdot \frac{\partial g_{i}}{\partial \mathrm{th}_{k}}+2 \sum_{i=0}^{N-1} \frac{\partial^{2} g_{i}}{\partial y_{i} \partial \mathrm{th}_{k}}
\end{aligned}
$$

The Formula (5) into the Formula (8) to solve partial derivative is:

$$
\begin{aligned}
& \frac{\partial g_{i}}{\partial \mathrm{th}}= \frac{\partial \eta}{\partial \mathrm{th}} \\
&= \begin{cases}-0.5 \cdot \operatorname{sgn}(y) \cdot m & |y|>\mathrm{th} \\
-0.5 \cdot \operatorname{sgn}(y) \cdot m \cdot \frac{|y|^{m+1}}{\mathrm{th}^{m+1}},|y| \leq \mathrm{th}\end{cases} \\
& \frac{\partial^{2} g_{i}}{\partial y_{i} \partial t h}= \begin{cases}0, & |y|>\mathrm{th} \\
-0.5 \cdot \operatorname{sgn}(y) \cdot n(m+1) & \frac{|y|^{m}}{\mathrm{th}^{m+1}}, \\
& |y| \leq \mathrm{th}\end{cases}
\end{aligned}
$$

In addition, we need to select the initial value of Newton's method. Initial value should be set to comply with its convergence condition. For the signal sequence which length is $N$, the universal threshold value can be selected as the initial threshold value. The termination condition of the iteration is $\left|\mathrm{th}_{\mathrm{k}+1}-\mathrm{th}_{\mathrm{k}}\right|<10^{-5}$ or $k<500$. 
By using the Newton's method to solve the $R(\mathrm{th})$ on the different decomposition scales, we can obtain the optimal threshold value $\mathrm{th}^{j}$ on the corresponding scale.

\section{De-Noise Simulation}

In order to verify the effect of de-noise algorithm presented in this paper. Adopts Matlab for simulation experiment. Add the white Gaussian noise to the heavy sine signal and the bumps signal. Then select the soft threshold function, the hard threshold function and the compromise threshold function to deal with the signal, compare these results with the method of this paper.
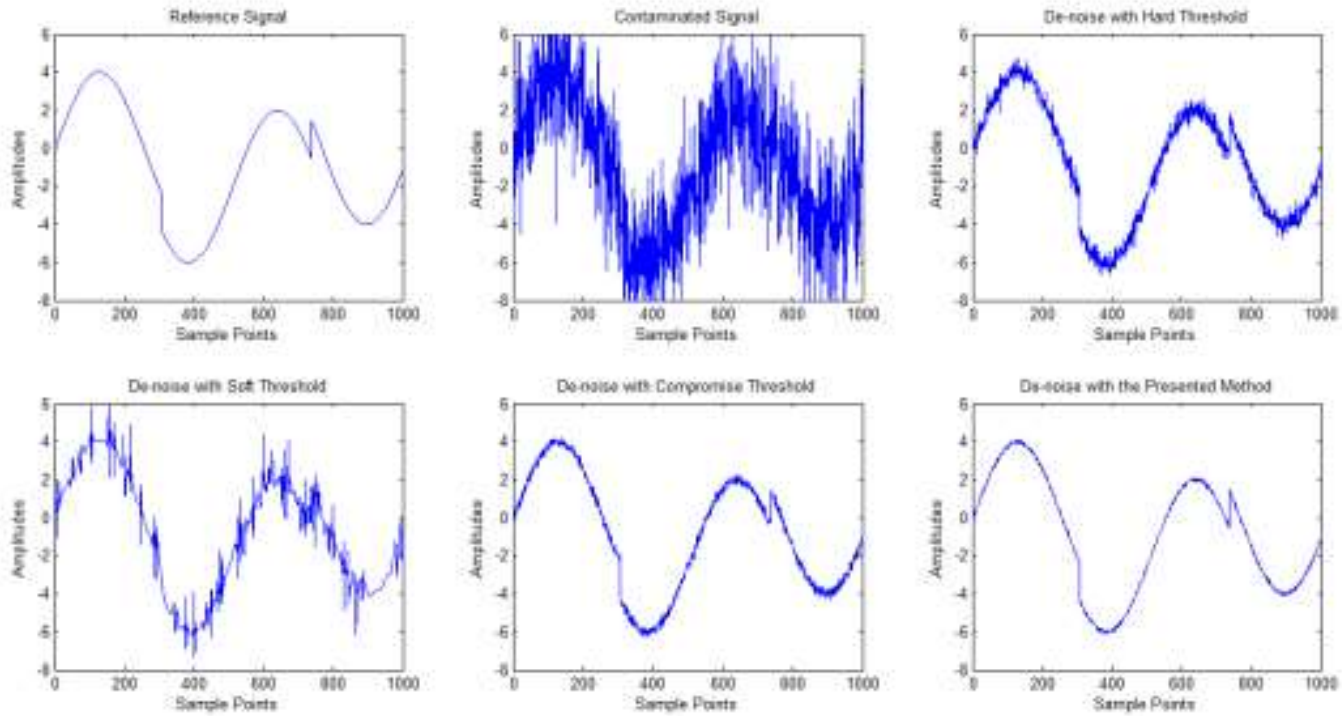

Figure 3. The Comparison Chart of Heavy Sine Signal De-Noise

Figure 3, is the effect figure of the heavy sine signal de-noises. Because of space constraints, the effect figure of the bumps signal de-noise is not given here.

Can be seen from the Figure 3, compared to the traditional threshold de-noise algorithm, the de-noise algorithm which is proposed in this paper can reach the desired objectives, and this algorithm will not reduce the detail of signal.

In order to objectively evaluate the effect of de-noise. The SNR, MSE and local peak error(LPE) are used as the indicator to measure the effect of de-noising. SNR is a measure of the effect of de-noising. MSE represents the similarity of the real signal and processed signal. LPE can be used to measure the extent of signal details missing.

Table 1, and Table 2, were de-noising results for heavy sine signal and bumps signal.

Table 1. The Result of Heavy Sine Signal De-Noise

\begin{tabular}{|c|c|c|c|c|}
\hline $\begin{array}{l}\text { de-noise } \\
\text { method }\end{array}$ & $\begin{array}{l}\text { hard threshold } \\
\text { de-noise }\end{array}$ & $\begin{array}{l}\text { soft threshold } \\
\text { de-noise }\end{array}$ & $\begin{array}{l}\text { compromise } \\
\text { threshold } \\
\text { de-noise }\end{array}$ & $\begin{array}{c}\text { The de-noise } \\
\text { method in this } \\
\text { paper }\end{array}$ \\
\hline $\mathrm{SNR}(\mathrm{dB})$ & 17.1287 & 16.8593 & 20.6418 & 21.9659 \\
\hline MSE & 0.1927 & 0.2269 & 0.0742 & 0.0495 \\
\hline $\operatorname{LPE}(\%)$ & 4.92 & 3.73 & 2.81 & 1.96 \\
\hline $\begin{array}{l}\text { de-noise } \\
\text { method }\end{array}$ & $\begin{array}{l}\text { hard threshold } \\
\text { de-noise }\end{array}$ & $\begin{array}{l}\text { soft threshold } \\
\text { de-noise }\end{array}$ & $\begin{array}{l}\text { compromise } \\
\text { threshold } \\
\text { de-noise }\end{array}$ & $\begin{array}{c}\text { The de-noise } \\
\text { method in this } \\
\text { paper }\end{array}$ \\
\hline $\mathrm{SNR}(\mathrm{dB})$ & 13.2956 & 12.9371 & 16.5794 & 18.2369 \\
\hline MSE & 0.1384 & 0.1057 & 0.0639 & 0.0407 \\
\hline
\end{tabular}




$\begin{array}{lllll}\operatorname{LPE}(\%) & 3.85 & 3.02 & 2.35 & 1.39\end{array}$

It can be seen from the table, when using this de-noise method in this paper, three indicators are better than other de-noise method.

\section{Conclusion}

For the problems of traditional wavelet de-noising algorithm may destroy the detail component of signal. This paper constructed a threshold function with parameter. This threshold function can be adaptively adjusting the parameters based on the energy of noise signal in the corresponding wavelet scale. Thus get optimal threshold function in the corresponding decomposition scales. And adopt Newton's method to obtain the optimal threshold. The results obtained in the simulation were as follows:

(1)When the signal is processed by wavelet de-noise, select the different threshold function will have different effects on the details of the signal. It can be concluded that the adaptive threshold function is superior to the traditional soft and hard threshold function by application LPE as evaluation index.

(2)Aiming at the effect of de-noise, adaptive wavelet de-noising is more targeted. Adaptive wavelet thresholding algorithm can adjust the threshold function and threshold based on the proportion of the noise in district wavelet decomposition scale.

(3)Except the threshold function and threshold may affect the effect of wavelet de-noise, The Wavelet also have some impact on the effect of de-noise.

\section{References}

[1] N. Mehdi and N. Hossein, "Image de-noising in the wavelet domain using a new adaptive thresholding function", Neurocomputing, vol. 72, no. 4/6, (2009), pp. 1012-1025.

[2] Z. Liangyu, W. Wanzhen and Z. Di, "A new method for the detection of the signal by using the wavelet de-noising method for the composite materials of aircraft", Journal of Harbin University of Science and Technology, vol. 06, (2014), pp. 27-31.

[3] X. P. Zhang and M. D. Desai, "Adaptive de-noising based on SURE risk", IEEE Signal Processing Letters, vol. 5, no. 10, (1998), pp. 265-267.

[4] W. Guangwen, J. Wang, D. Bao, C. Yong and H. Yangpo, "De-noising based on adaptive threshold function of wavelet threshold de-noising method", Journal of electronics and information technology, vol. 06, (2014), pp. 1340-1347.

[5] Y. Zongna and W. Lianxin, "Adaptive wavelet threshold de-noising new threshold function based on ", Technology, vol. 11, (2014), pp. 12-15+20.

[6] W. Zhiqiang, "A de-noising algorithm", Journal of Harbin University of Science and Technology new wavelet threshold function based on vol. 04, (2011), pp. 56-58.

[7] P. Quan, M. Jinli and Z. Lei, Journal of [J]. Wavelet filtering method and application of electronic and information, vol. 29, no. 1, (2007), pp. 236-242.

[8] M. Jinli, Pa. Quan and Z. Hongcai, "The multi-scale product coefficients of semi soft threshold wavelet filter", Journal of electronics and information technology, based on the vol. 29, no. 7, (2007), pp. 1649-1652.

[9] X. Mingyi, "To eliminate the noise of [J]. Sudden three-phase short circuit test data of Harbin University of Science and Technology wavelet algorithm, vol. 02, (2011), pp. 118-121.

[10] Y. P. Gao, T. Zhaosheng, "mild Kaiser window interpolation FFT harmonic analysis and application", China Motor Engineering Journal of, vol. 30, no. 4, (2010), pp. 43-48.

[11] L. Xinxin, "Feature extraction of acoustic signals from ship based on Hilbert Huang transform", Journal of Harbin University of Science and Technology, vol. 03, (2014), pp. 69-73. 